\title{
The mechanism of adenosine-mediated activation of IncRNA MEG3 and its antitumor effects in human hepatoma cells
}

\author{
LI-XUAN LIU ${ }^{1}$, WEI DENG ${ }^{1}$, XIAO-TAO ZHOU ${ }^{1}$, RUI-PEI CHEN ${ }^{1}$, MENG-QI XIANG $^{1}$, \\ YI-TIAN GUO ${ }^{1}$, ZE-JIN PU ${ }^{1}$, RUI LI ${ }^{2}$, GE-FEI WANG ${ }^{3}$ and LING-FEI WU ${ }^{1}$ \\ ${ }^{1}$ Department of Gastroenterology, Second Affiliated Hospital, ${ }^{2}$ Key Laboratory of Infectious Diseases and \\ Immunopathology of Guangdong Province, ${ }^{3}$ Department of Microbiology and Immunology, \\ Shantou University Medical College, Shantou, Guangdong 515041, P.R. China
}

Received October 13, 2015; Accepted November 30, 2015

DOI: $10.3892 /$ ijo.2015.3248

\begin{abstract}
Long non-coding RNA MEG3 is suggested to function as a tumor suppressor. However, the activation mechanism of MEG3 is still not well understood and data are not available on its role under adenosine-induced apoptosis. In this study, HepG2 cells were treated with adenosine or 5-Aza-cdR. Methylation status of MEG3 promoter was detected by methylation specific PCR (MSP) and MEG3 expression was determined by qRT-PCR. PcDNA3.1-MEG3 recombinant plasmid was constructed and transfected to hepatoma HepG2 and Huh7 cells. Cell growth, morphological changes, cellcycle distribution and apoptosis were analyzed by MTT assay, fluorescence microscopy and flow cytometry. The mRNA and protein expression levels were detected by qRT-PCR and western blot analysis. MEG3 binding proteins were screened by the improved MS2 biotin tagged RNA affinity purification method. The co-expression network of MEG3 was generated by GO analysis and ILF3 was identified as MEG3 binding protein by RNA pulldown and western blot analysis. Both adenosine and 5-Aza-CdR increased MEG3 mRNA expression and the CpG island of MEG3 gene in HepG2 cells was typical hypermethylation. Ectopic expression of MEG3 inhibited hepatoma cell growth in a time-dependent manner, resulted in cell cycle arrest and induced apoptosis. Ectopic expression of MEG3 increased p53, caspase-3 mRNA and protein levels, decreased MDM2 and cyclin D1 mRNA and protein levels, as well as ILF3 protein expression in HepG2 cells. These findings are the first to identify that adenosine increases MEG3 expression by inhibition of DNA methylation and its antitumor effects is involved in MEG3 activation. ILF3 may participate in the anticancer regulation of MEG3 by interacting with MEG3.
\end{abstract}

Correspondence to: Dr Ling-Fei Wu, Department of Gastroenterology, Second Affiliated Hospital, Shantou University Medical College, Shantou, Guangdong 515041, P.R. China

E-mail: 1808435253@qq.com

Key words: lncRNA MEG3, hepatocellular carcinoma, DNA methylation, adenosine, ILF3, apoptosis, p53, MDM2s

\section{Introduction}

Hepatocellular carcinoma (HCC) is the most common cancer and the third leading cause of cancer-related deaths worldwide (1). Yet, the pathogenesis mechanisms of HCC remain unclear. One of the potential molecular mechanisms involves the long non-coding RNAs (lncRNAs) $(2,3)$.

LncRNAs play an important role in tumorigenesis and investigation of their biological functions and molecular mechanisms is important for understanding the development and progression of cancer (4). The IncRNA HOTAIR is overexpressed in HCC tissues compared to corresponding non-cancerous tissues and high HOTAIR expression tightly correlated with the presence of liver metastasis (5). IncRNA maternally-expressed gene 3 (MEG3) is an imprinted gene, located on human chromosome $14 q 32.3$ (6). The downregulation of MEG3 has been observed in various human cancers (7-9). MEG3 might function as a tumor suppressor $(10,11)$. However, the reasons for its downregulation and its function in HCC are not well understood.

Adenosine and their analogues can interfere with the synthesis of nucleic acids and exert genotoxic activity by incorporating with and altering the DNA or RNA macromolecules, which makes them exciting candidates for anticancer therapy (12-14). Although MEG3 plays a role in inhibiting tumor growth, no data are available on its function under genotoxic stress-induced apoptosis. In our previous study, we demonstrated that adenosine induces hepatoma cell apoptosis and the mechanism is involved in DNA demethylation and genotoxic effects $(15,16)$. In this study, we treated HepG2 cells with adenosine or 5-Aza-2'-deoxy-cytidine (5-Aza-CdR), a selective DNA methyltransferase inhibitor, and observed the methylation status of MEG3 promoter and MEG3 mRNA expression. In addition, an improved MS2-BioTRAP (MS2 biotin tagged RNA affinity purification) technique was applied to screen MEG3-binding protein in HEK293 cell line. The antitumor effect of adenosine and MEG3 in HCC was observed and the possible molecular mechanisms were investigated.

\section{Materials and methods}

Cell culture and experimental groups. HepG2, Huh-7 and HEK293 cells, obtained from the American Type Culture 
Collection, were cultured in Dulbecco's modified Eagle's medium supplemented with $10 \%(\mathrm{v} / \mathrm{v})$ fetal bovine serum, penicillin (final concentration, $100 \mathrm{U} / \mathrm{ml}$ ), and streptomycin (final concentration, $100 \mathrm{mg} / \mathrm{ml}$ ) under a humidified atmosphere of $5 \% \mathrm{CO}_{2}$ and $95 \%$ air at $37^{\circ} \mathrm{C}$. Cells were passaged at $70-80 \%$ confluence. HCC cells were divided into two groups for the experiment of overexpression MEG3: pcDNA3.1 (control group) and pcDNA3.1-MEG3 vector (experimental group).

Treatment of HepG2 cells with adenosine or 5-Aza-CdR. A total of $1.0 \times 10^{5} \mathrm{HepG} 2$ cells were inoculated into each well of a 6 -well plate. After the cells attached, the original medium was replaced with fresh medium containing $4.0 \mathrm{mmol} / 1$ adenosine for $48 \mathrm{~h}$ or $20 \mu \mathrm{mol} / 15$-Aza-cdR for $72 \mathrm{~h}$. The medium with the same concentration of 5-Aza-CdR was replaced daily. Cells were collected and prepared for detection of MEG3 expression.

Detection of DNA methylation by methylation-specific PCR $(M S P)$. A total of $1.0 \times 10^{5} \mathrm{HCC}$ cells were inoculated into each well of a 6-well plate and treated as described above (16). DNA from the cells was extracted using an EZNA Tissue DNA kit according to the manufacturer's instructions. The methylation status of the MEG3 gene was determined by MSP assay. For the MSP, the CpGenome Turbo Bisulfite Modification kit was used in accordance with the manufacturer's instructions. The sequences of the methylation-specific primers of MEG3 were as follows: the methylated pair (M), 5'-GTTAGTAATCGGG TTTGTCGGC (forward) and 5'-AATCATAACTCCGAACAC CCGCG (reverse); the unmethylated pair(U),5'-GAGGATGGT TAGTTATTGGGGT (forward) and 5'-CCACCATAACCA ACACCCTATAATCACA (reverse). MEG3 DNA was amplified in a DNA Engine Peltier thermal cycler (Bio-Rad) by 1 cycle of $95^{\circ} \mathrm{C}$ for $15 \mathrm{~min}$ followed by 5 cycles of $94^{\circ} \mathrm{C}$ for $30 \mathrm{sec}, 70^{\circ} \mathrm{C}$ for $30 \mathrm{sec}$ and $72^{\circ} \mathrm{C}$ for $30 \mathrm{sec} ; 5$ cycles of $94^{\circ} \mathrm{C}$ for $30 \mathrm{sec}, 65^{\circ} \mathrm{C}$ for $30 \mathrm{sec}$ and $72^{\circ} \mathrm{C}$ for $30 \mathrm{sec} ; 30$ cycles of $94^{\circ} \mathrm{C}$ for $30 \mathrm{sec}, 60^{\circ} \mathrm{C}$ for $30 \mathrm{sec}$ and $72^{\circ} \mathrm{C}$ for $30 \mathrm{sec}$; and 1 cycle of $72^{\circ} \mathrm{C}$ for $7 \mathrm{~min}$. The PCR products were identified by electrophoresis through a $2.0 \%$ agarose gel and ethidium bromide staining.

Plasmid identification and cell transfection and bacterial transformation. PcDNA3.1-MEG3 plasmid was constructed as previously described (16). PcDNA3.1-MEG3 plasmids were extracted from $E$. coli according to the manufacturer's specifications. Both restriction enzymes, XhoI and BamHI, were used to cut the pcDNA3.1-MEG3 plasmid DNA for $1 \mathrm{~h}$ at $37^{\circ} \mathrm{C}$. Subsequently, DNA fragments after digestion were identified by the agarose gel electrophoresis and quantified by capturing the ethidium bromide absorbance with a fluorescence microscope (Olympus BX51, Tokyo, Japan).

For ectopic MEG3 expression, the full-length MEG3 cDNA was subcloned into the pcDNA3.1 plasmid vector and transfected into HepG2 or $\mathrm{HuH} 7$ cells as previously described (16). PcDNA3.1 was transfected to cells in parallel as a control. The cells were then subjected to RNA/protein extraction and further functional assays.

RNA extraction and qRT-PCR analysis. HepG2 and Huh7 cells were transfected with pcDNA3.1-MEG3 vector or
Table I. Primer sequences for quantitative real-time PCR.

\begin{tabular}{ll} 
Gene & \multicolumn{1}{c}{ Primer sequences } \\
\hline MEG3 & F: 5'-CTCAGGCAGGATCTGGCATA-3' \\
& R: 5'-CCTGGAGTGCTGTTGGAGAA-3' \\
p53 & F: 5'-TGAAGCTCCCAGAATGCCAG-3' \\
& R: 5'-GGGAGTACGTGCAAGTCACA-3' \\
MDM-2 & F: 5'-ATCAGGCAGGGGAGAGTGAT-3' \\
& R: 5'-CAATTCTCACGAAGGGCCCA-3' \\
Caspase-3 & F: 5'-AGAGCTGGACTGCGGTATTGGAG-3' \\
& R: 5'-GAACCATGACCCGTCCCTTG-3' \\
Cyclin D1 & F: 5'- GTGCATCTACACCGACAACTCCA-3' \\
& R: 5'- GTGCATCTACACCGACAACTCCA-3' \\
18S & F: 5'-AAACGGCTACCACATCCAAG-3' \\
& R: 5'-CAATTACAGGGCCTCGAAAG-3' \\
\hline
\end{tabular}

pcDNA3.1 for $48 \mathrm{~h}$. The total RNA was isolated with TRIzol reagent, according to the manufacturer's protocol. For reverse transcriptase analysis, $1 \mu \mathrm{g}$ of total RNA was reverse-transcribed using random primers under standard conditions using an EasyScript First-Strand cDNA Synthesis Super Mix kit. Real-time PCR amplification with one microliter of the reverse transcriptase reaction mixture was performed on the ABI 7500 real-time PCR system (Applied Biosystems, Foster City, CA, USA) using SYBR Premix Ex Taq following the manufacturer's instructions. PCR conditions were $95^{\circ} \mathrm{C}$ for $30 \mathrm{sec}$, followed by 40 cycles of $95^{\circ} \mathrm{C}$ for $3 \mathrm{sec}$ and $60^{\circ} \mathrm{C}$ for $30 \mathrm{sec}$. Gene-specific primers are listed in Table I. 18s rRNA was used as a housekeeper gene for the qRT-PCR reactions. Fold change of MEG3, p53, MDM2, caspase- 3 and cyclin D1 was calculated by the $2^{-\Delta \Delta C t}$ method.

Protein extraction and western blot analysis. Cells were washed with ice-cold PBS, harvested and lysed in RIPA buffer supplemented with protease inhibitor cocktail. Cell lysates were centrifuged at $12,000 \mathrm{rpm}$ at $4^{\circ} \mathrm{C}$ for $10 \mathrm{~min}$. The supernatant was boiled for $5 \mathrm{~min}$ and subjected to $12 \%$ SDS-PAGE and transferred to a nitrocellulose membrane. The membranes were blocked with $5 \%$ fat-free milk for $1 \mathrm{~h}$ at room temperature, followed by incubation with primary antibodies against $\beta$-actin $(1: 3,000), \operatorname{Mdm} 2$ (1:500), cyclin D1 (1:500), total caspase-3 (1:800), cleaved caspase-3 (1:800) and ILF3 $(1: 1,000)$ at $4^{\circ} \mathrm{C}$ overnight. The next day, the membranes were incubated with biotin-conjugated secondary antibody for $1 \mathrm{~h}$ at room temperature, followed by incubation with an HRP complex at room temperature for $30 \mathrm{~min}$. Bands were visualized with an ECL detection system (Thermo Scientific, Waltham, MA, USA). Protein expression was analyzed by the Quantity One software (Bio-Rad, Hercules, CA, USA) and normalized to that of $\beta$-actin.

Identification of proteins associated with lncRNA MEG3. An improved MS2-BioTRAP (MS2 biotin tagged RNA affinity purification) technique was used which relies on the interaction of the bacteriophage MS2 coat-protein with a specific viral 
A

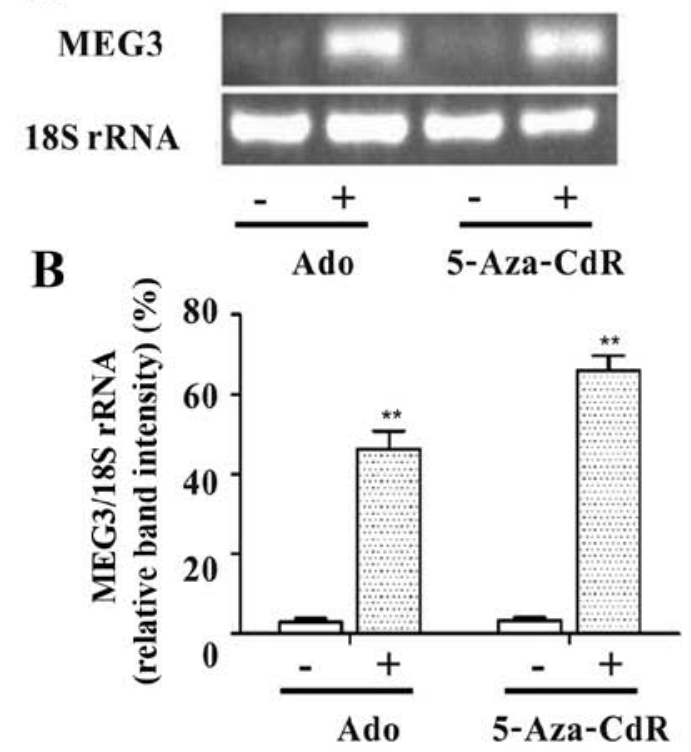

C

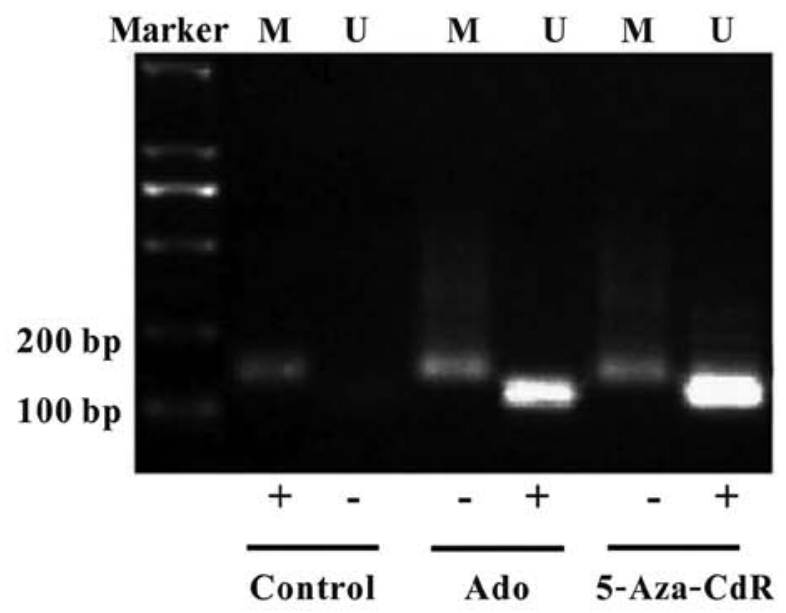

Figure 1. Methylation status of MEG3 promoter in HepG2 cells. (A) The mRNA expression of MEG3 in HepG2 cells treated with adenosine or 5-Aza-CdR by RT-PCR assay. (B) Both adenosine and 5-Aza-CdR significantly increased MEG3 mRNA expression. (C) CpG island methylation status was detected by MSP assay. For each sample a primer set for the methylated (M) and unmethylated (U) copies of the MEG3 gene was used. A 160-bp product represents the methylated state and a 120-bp PCR product represents the unmethylated state. Data are presented as the means \pm SD. Independent experiments were repeated three times. ${ }^{*} \mathrm{p}<0.05$ vs. control; ${ }^{* *} \mathrm{p}<0.01$ vs. control.

RNA stem-loop structure that was inserted into or grafted onto the $3^{\prime}$ end of the IncRNA MEG3 (17). Twelve copies of the MS2 coat-protein binding site were introduced into the region encoding the MEG3 to generate a MEG3-MS2bs expression plasmid. This expression plasmid and a second plasmid encoding a Flag-tagged MS2 coat-protein were transiently introduced into cultured HEK293 cells. In brief, the pcDNA3.1-Flag-MS2 vector, pcDNA3.1-MS2bs vector and pcDNA3.1-MEG3-MS2bs vector were constructed as previously described by us (16). HEK293 cells are cultured and harvest at $80 \%$ confluence, followed by co-transfection with pcDNA3.1-Flag-MS2 vector and pcDNA3.1-MEG3MS2bs using Lipofectamine 2000. Cells co-transfected with pcDNA3.1 and pcDNA3.1-MEG3-MS2bs vector were used as a control. Cells were further incubated for $48 \mathrm{~h}$, then collected for RIP experiments. The protein components were determined by mass spectrometry, and the candidate proteins were confirmed by western blot analysis.

RNA binding protein immunoprecipitation. HEK293 cells were harvested and lysed in RIP lysis buffer with protein inhibitor, RNA inhibitor and DNase I on ice. Cell lysates were preincubated with the antibody. RNAs were immunoprecipitated with the antibody against RBP and protein $A / G$ magnetic beads. The magnetic bead bound complexes were immobilized with a magnet and unbound material was washed off. Then, RNAs were extracted, precipitated and turned into a cDNA library by ImProm-II reverse Transcription system (Promega, USA). The remaining beads were boiled and separated by SDS-PAGE gel for western blot analysis.

Statistical analysis. All the experiments described were performed at least in triplicate. The data are expressed as mean \pm SD. Statistical significance was calculated using either the Student's t-test, or one-way analysis of variance (ANOVA) with a post-hoc test of multiple comparisons. A p $<0.05$ was considered statistically significant.

\section{Results}

Downregulation of MEG3 is due to hypermethylation in the MEG3 promoter region. As shown in Fig. 1A and B, the mRNA relative expression level of MEG3 significantly increased after treatment with adenosine or 5-Aza-CdR (Fig. 1A and B). The unmethylated pattern (U) and a methylated pattern $(\mathrm{M})$ were observed in the cells treated with adenosine or 5-Aza-CdR by MSP. The two DNA methyltransferase inhibitors, which reverse the abnormal methylation status of the MEG3 promoter, increased the expression of unmethylated product (120 bp, Fig. 1C). The results indicate that the methylation status of MEG3 promoter may lead to downregulation of MEG3 expression in HepG2 cells.

PcDNA3.1-MEG3 plasmid is constructed and identified. PcDNA3.1-MEG3 plasmid that expressed lncRNA MEG3 was constructed. LncRNA MEG3 gene was inserted into the pcDNA3.1 vector, which was confirmed by XhoI and BamHI restriction enzyme digestion. The particular sequence of MEG3 insertion into vectors was the same as designed and the fragment was not present in the control vector, showing pcDNA 3.1-MEG3 plasmid was successfully constructed (Fig. 2).

Ectopic expression of MEG3 and its effects on cell growth inhibition. HepG2 and Huh7 cells were transiently transfected with either pcDNA3.1 (control) or pcDNA3.1-MEG3, and MEG3 mRNA expression was measured by qRT-PCR. Compared to the control group, MEG3 mRNA expression 


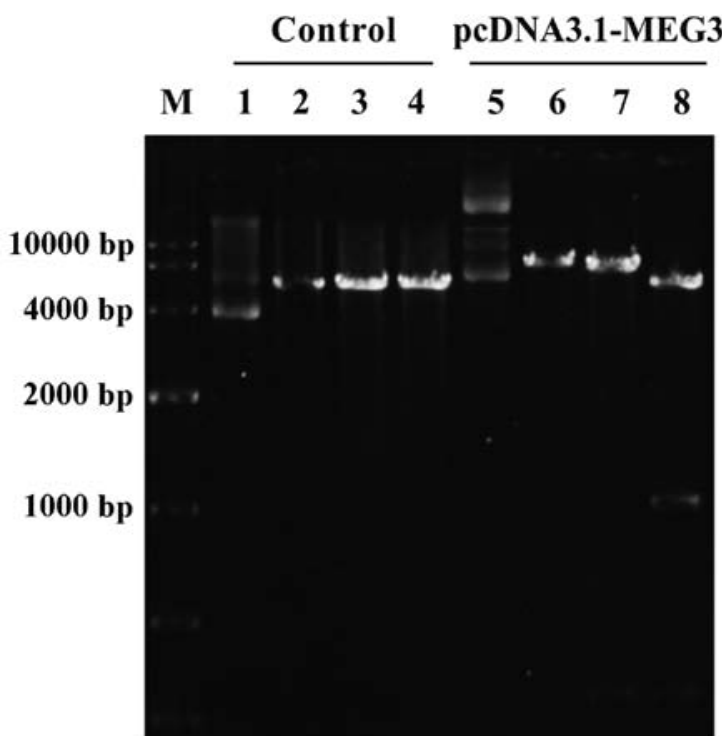

Figure 2. Identification of the MEG3 gene by restriction enzyme digestion in pcDNA3.1 or pcDNA3.1-MEG3 plasmids. PcDNA3.1 or pcDNA3.1-MEG3 plasmids were constructed and extracted from E. coli according to the manufacturer's specifications. Restriction enzymes ( $\mathrm{XhoI}$ and $\mathrm{BamHI})$ were used for $1 \mathrm{~h}$ at $37^{\circ} \mathrm{C}$ to cut the plasmid DNA. DNA fragments after digestion were identified by the agarose gel electrophoresis and quantified by capturing the EB absorbance under a fluorescence microscope. Lane M, DNA marker: lanes 1-4, restriction enzyme digestion of pcDNA3.1; lanes 5-8, restriction enzyme digestion of pcDNA3.1-MEG3. Lanes 1 and 5, uncut plasmid; lanes 2 and 6, XhoI digestion; lanes 3 and 7, BamHI digestion; lanes 4 and 8 , XhoI+BamHI digestion. The particular sequence of MEG3 was cut to two fragments in lane 8 . levels increased by $>90$-fold or 80 -fold in MEG3-transfected HepG2 or Huh7 cells (Fig. 3A), showing the high level mRNA expression of MEG3 was obtained in cells.

The effect of MEG3 on the proliferation of hepatoma cells was measured using the MTT assay. Ectopic expression of MEG3 significantly decreased cell vitality rate by 29.6 and $31.2 \%$ after 48 and $72 \mathrm{~h}$ in HepG2 cells, respectively, compared with the control group ( $\mathrm{p}<0.01)$. Ectopic expression of MEG3 significantly decreased cell vitality rate by 31.5 and $45.9 \%$ after 48 and $72 \mathrm{~h}$ in Huh7 cells, respectively, compared with the control group (Fig. 3B, p<0.01). These results suggested that ectopic expression of MEG3 can inhibit tumor cell proliferation.

MEG3 induces cell apoptosis and cell cycle arrest in vitro. To detect whether apoptosis was a contributing factor to cell growth inhibition, hepatoma cells were stained with Hoechst 33258 after transfection with plasmids. The nuclei of the control group were uniform and without condensation or fragmentation. Compared with the control group (pcDNA3.1), nuclei in cells with ectopic expression of MEG3 became condensed and shrunken, and typical apoptotic bodies appeared. The percentages of apoptosis cells after MEG3 plasmid transfection are 18.1 and $13.3 \%$ in HepG2 and Huh7 cells, respectively, which is significantly higher than that of the control group (Fig. 4C, p<0.01).

In order to further investigate whether MEG3-induced apoptosis is related to the cell cycle, the cell cycle distribu-
A

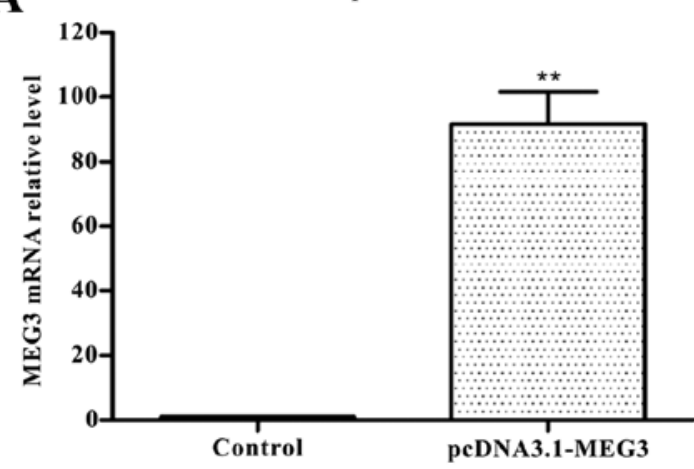

B

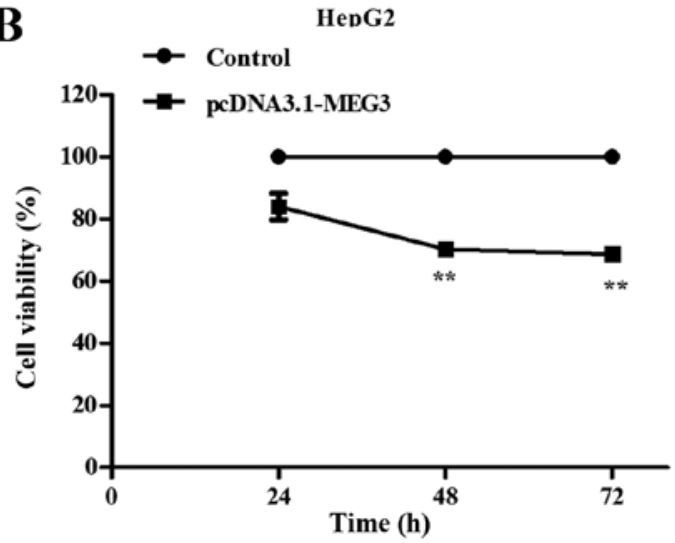

Huh7
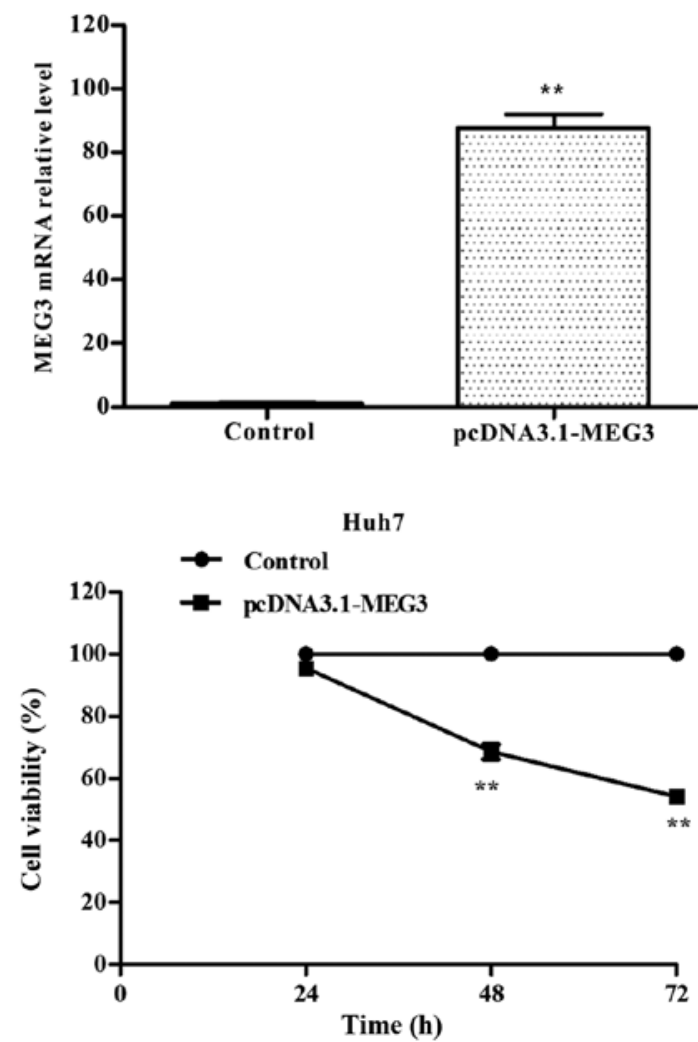

Figure 3. Ectopic expression of MEG3 and its effects on cell proliferation in hepatoma cells. (A) HepG2 and Huh7 cells were transiently transfected with either pcDNA3.1 (control) or pcDNA3.1-MEG3, and MEG3 mRNA expression was measured by qRT-PCR. (B) Cell viability was evaluated at different times by the MTT assay. Ectopic expression of MEG3 significantly inhibited hepatoma cell growth in a time-dependent manner. ${ }^{* *}$ p $<0.01$ vs. control. 
$\mathbf{A}$

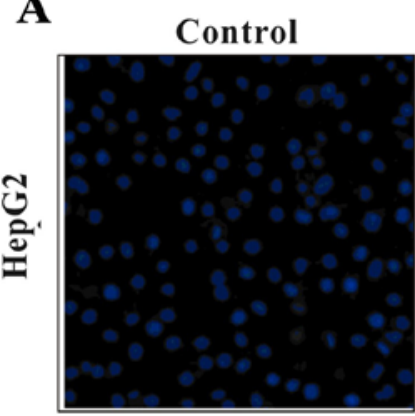

pcDNA3.1-MEG3
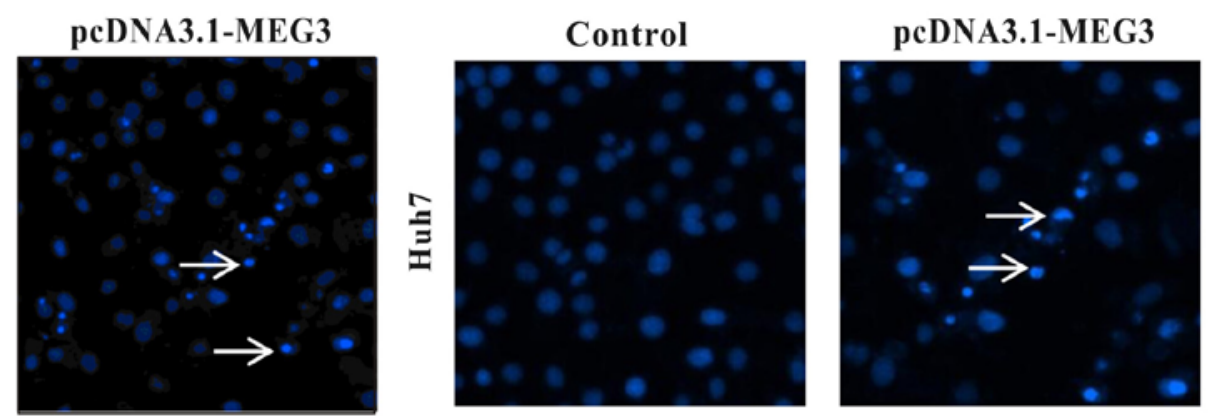

B

HepG2

\section{Huh7}

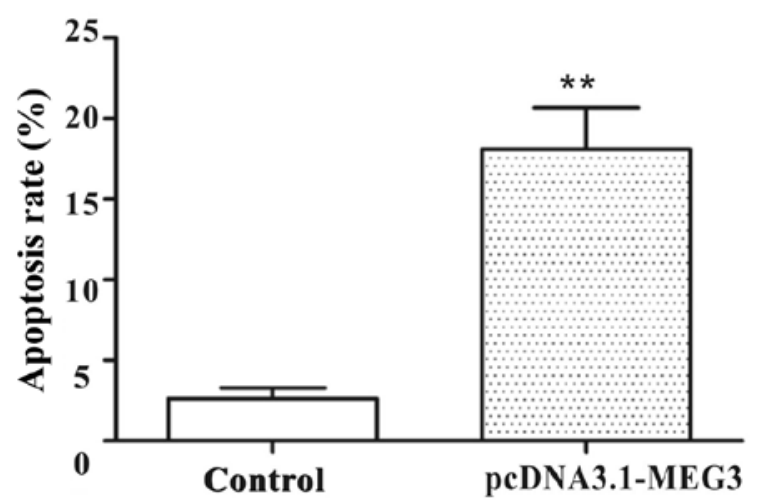

C

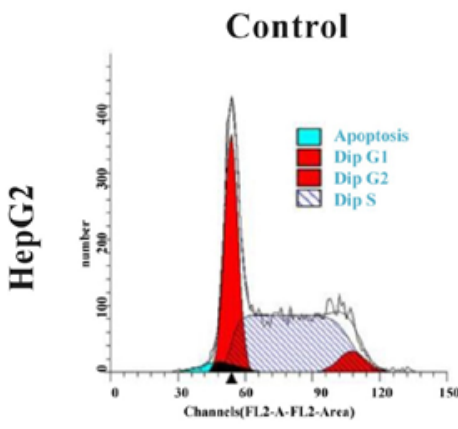

pcDNA3.1-MEG3

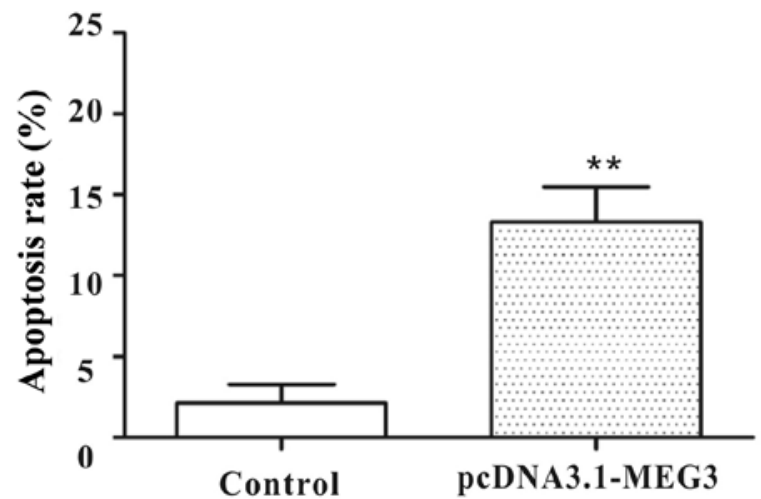

D
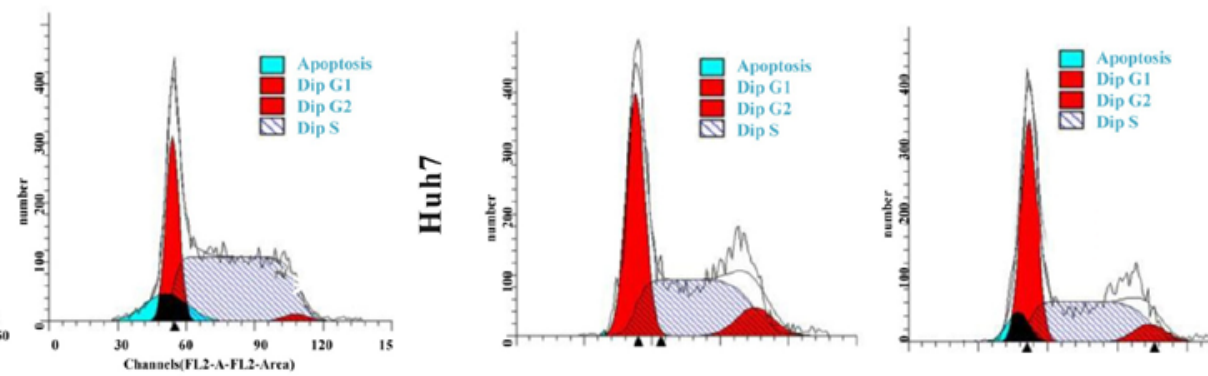

\section{Huh7}
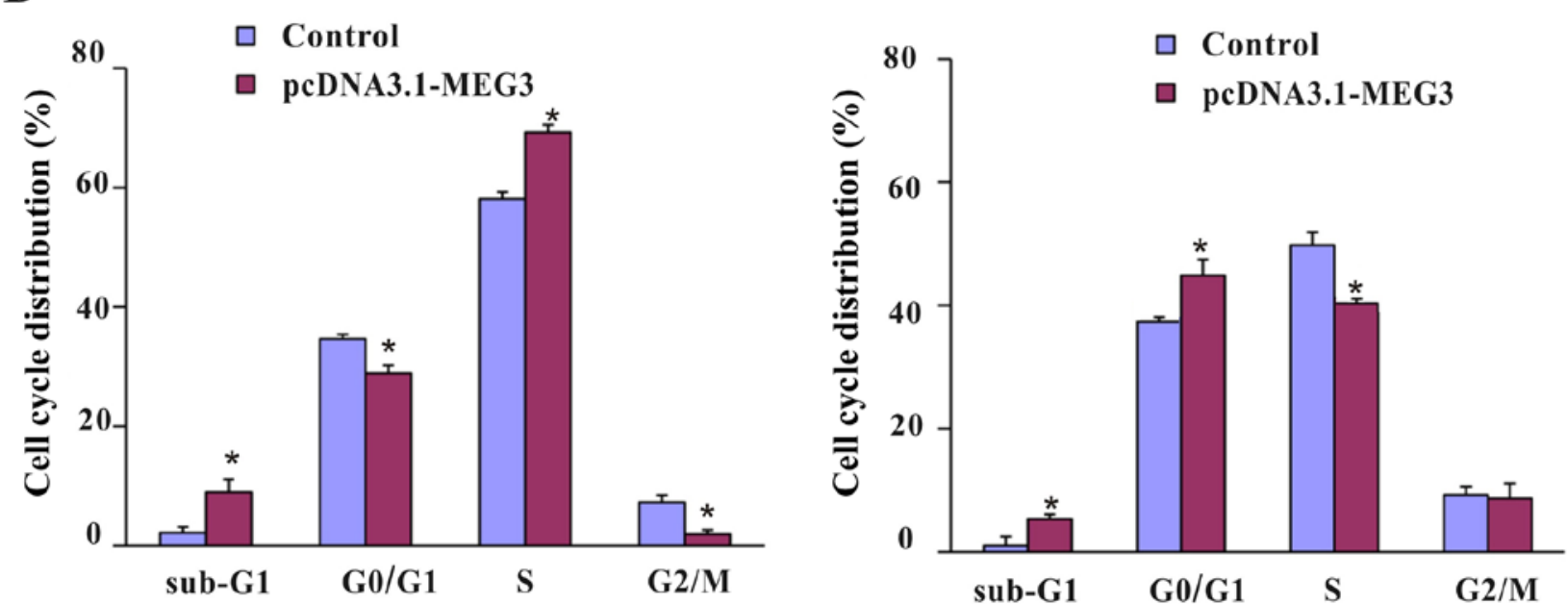

Figure 4. Effects of ectopic expression of MEG3 on apoptosis and cell cycles in the hepatoma cells. (A) The morphology of apoptotic nuclei was observed by Hoechst 33258 staining and fluorescence microscopy. The percentage of apoptotic nuclei per optical field was scored. (B) Quantitative analysis of the total apoptotic cells by fluorescence microscopy. Each bar corresponds to the mean $\pm \mathrm{SD}$ of three independent experiments. (C) Cell cycles and apoptosis percentage were analyzed by flow cytometry and the apoptosis cells in sub-G1 phase (blue peak) were determined. (D) Quantitative analysis of the cell cycles. Bar chart represents the percentage of cells in $\mathrm{G} 0 / \mathrm{G} 1, \mathrm{~S}$, sub-G1 or G2/M phase. ${ }^{*} \mathrm{p}<0.05$ vs. control; ${ }^{* *} \mathrm{p}<0.01$ vs. control. 
$\mathbf{A}$
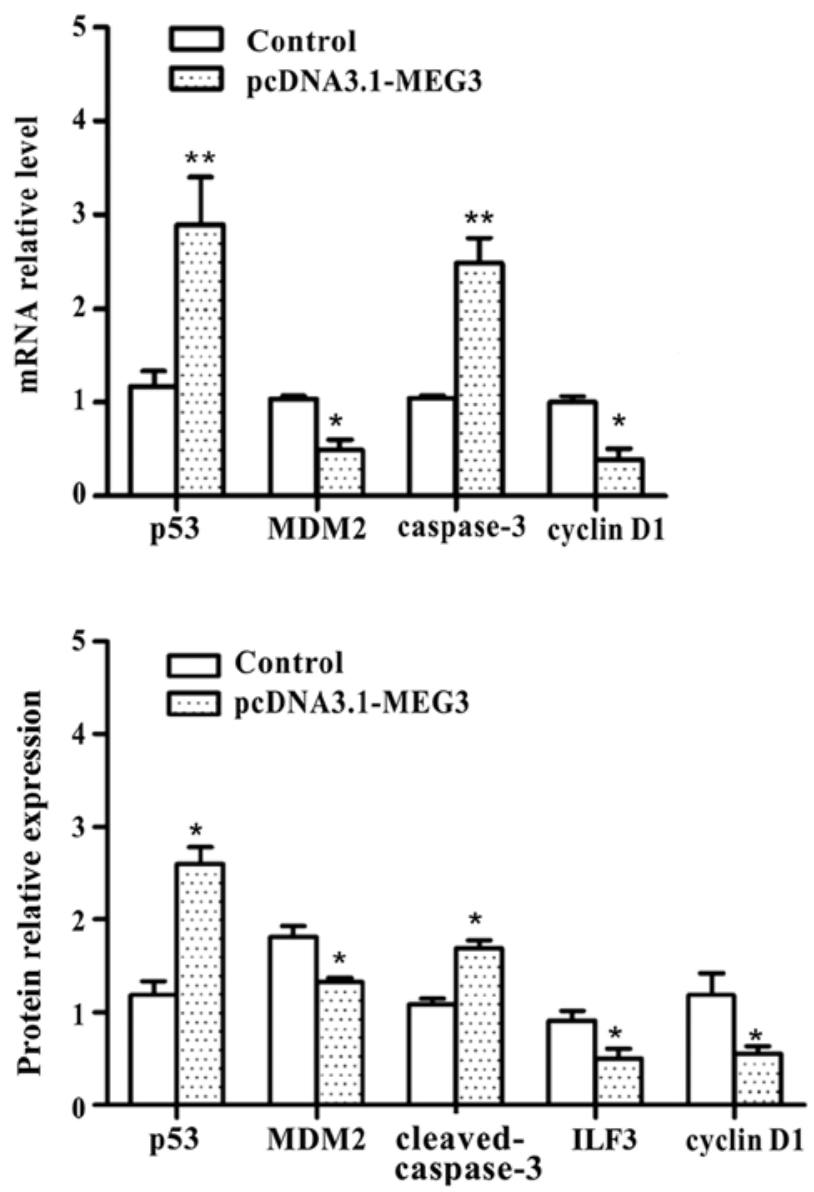

B

\section{Control pcDNA3.1-MEG3}

p53

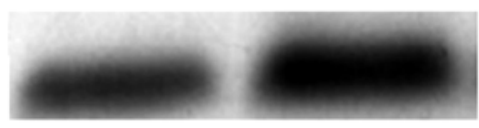

MDM2

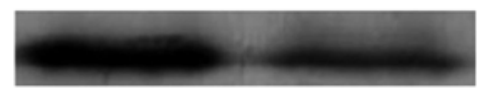

$\beta$-actin

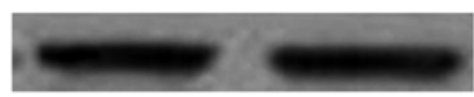

caspase-3

cleaved-

caspase-3

$\beta$-actin

ILF3

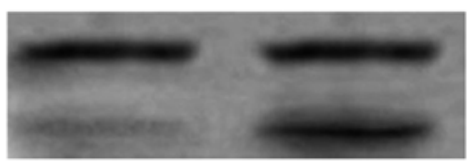

cyclin D1

$\beta$-actin
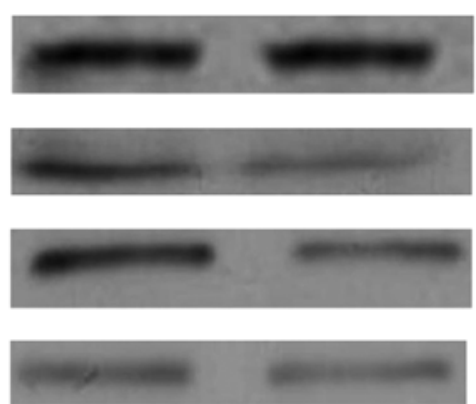

Figure 5. Effects of ectopic MEG3 on mRNA and protein expression of p53, MDM2, caspase-3, cyclin D1 and ILF3. HepG2 cells were transfected with either pcDNA3.1-MEG3 or pcDNA3.1 (control). (A) qRT-PCR was used to detect mRNA relative expression. (B) Western blot analysis was used to detect protein expression. (C) Quantitative analysis of the protein expression. Values represent the means \pm SD of three independent experiments. ${ }^{*} \mathrm{p}<0.05 \mathrm{vs}$. control; ** $<<0.01$ vs. control.

tion was detected by flow cytometry. Ectopic expression of MEG3 significantly decreased the cells of G0/G1 and G2/M phase, but increased the percentage of $\mathrm{S}$ phase from 58.08 to $69.27 \%$ in HepG2 cells. Ectopic expression of MEG3 significantly increased the percentage of $\mathrm{G} 0 / \mathrm{G} 1$ phase from 38.50 to $48.89 \%$ and decreased the percentage of $\mathrm{G} 2 / \mathrm{S}$ phase in Huh7 cells (Fig. 4C and D, p<0.05). Statistical results showed that ectopic expression of MEG3 increased the percentage of apoptotic cells (sub-G1 phase) in both cell types ( $<<0.05$, Fig. $4 \mathrm{D})$, which is consistent with the result of fluorescence microscopy observations (Fig. 4B). Taken together, these results confirm that cell growth inhibition and cell cycle arrest caused by MEG3 is facilitated by the induction of apoptosis.

MEG3-triggered apoptosis is mediated by $p 53$ pathway and cell cycle arrest. To probe the mechanism by which MEG3 initiated the cell cycle arrest, the investigation was focused on the cell cycle checkpoint, the cyclins (cyclin D1), p53 and other relative factors. Ectopic expression of MEG3 resulted in marked reductions in the mRNA and protein expression of cyclin D1 and MDM2. By contrast, the expression of p53 and caspase-3 mRNA and protein levels significantly increased in HepG2 cells (Fig. 5A-C, p<0.05). These data confirm that MEG3 functions as a tumor suppressor gene by activating the p53-mediated apoptosis pathway and causes cell cycle arrest in HepG2 cells.

ILF3 was also investigated by western blot analysis. The result showed that the expression level of ILF3 decreased after transfection with pcDNA3.1-MEG3, compared with the control group (Fig. 5B and C, p<0.05). The data indicated that ILF3 protein expression is negatively regulated by MEG3 in HepG2 cells.

Screening of MEG3-binding protein. MS2-BioTRAP (MS2 biotin tagged RNA affinity purification) experiment was carried out (Fig. 6). Sequence analysis and restriction endonuclease analysis of target genes demonstrated that the position and size of target gene cDNA insertion were consistent with the design (Fig. 6A-E). The pcDNA3.1-Flag-MS2 and pcDNA3.1-MEG3-MS2bs vectors were co-transfected into HEK293 cells for the RIP experiment. The expression intensity of MEG3 mRNA in co-transfected group with pcDNA3.1-Flag-MS2 and pcDNA3.1-MEG3-MS2bs is $\sim 98$-fold higher than that of the control group, showing it is an effective method to study RNA-protein interaction 


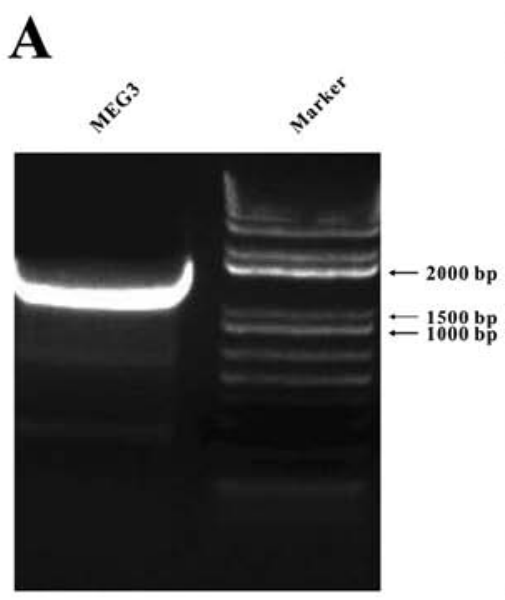

$\mathbf{F}$

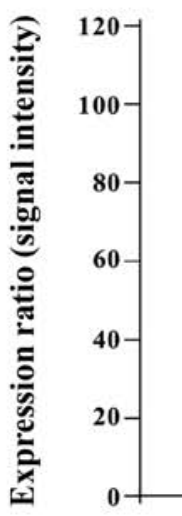

pcDNA 3.1
pcDNA 3.1-MS2bs peDNA 3.1-Flag $\times$ MS2

peDNA 3.1-MEG3-MS2bs
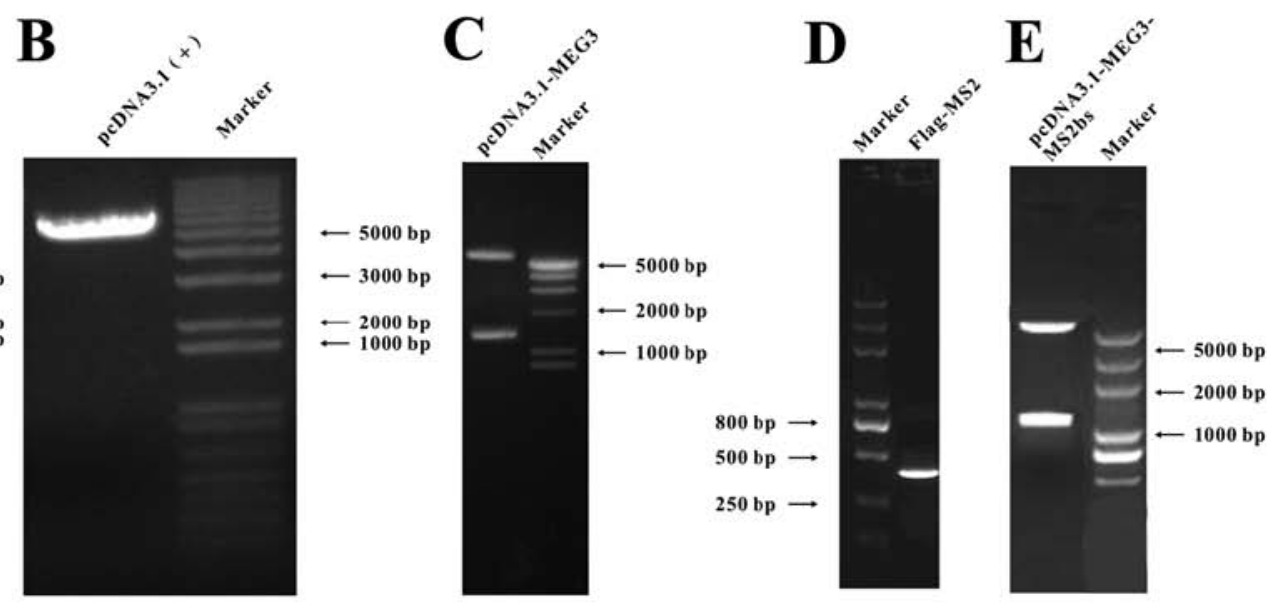

G

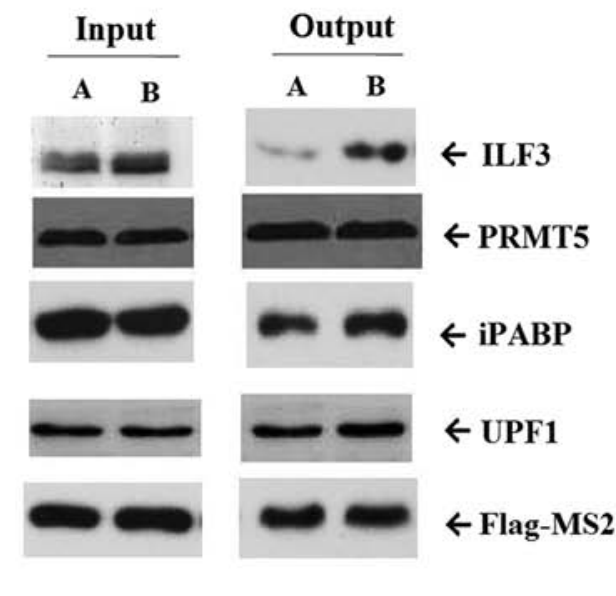

Figure 6. MS2-BioTRAP experiments and MEG3-binding protein screening. (A-E) Construction and identification of several products: MEG3 (A), pcDNA3.1 (B), pcDNA3.1-MEG3 (C), Flag-MS2 (D) and pcDNA3.1-MEG3-MS2bs (E). (F) Detection of MEG3 RNA in affinity purification of RNA-protein complexes by RT-PCR. (G) Verification of candidate MEG3 binding proteins by western blot analysis. The pcDNA3.1-Flag-MS2 and pcDNA3.1-MEG3MS2bs vectors were co-transfected into HEK293 cells (B group). The pcDNA3.1-Flag-MS2 and pcDNA3.1-MS2bs vector were co-transfected into HEK293 cells as control (A group). Cells were irradiated with $254 \mathrm{~nm}$ UV light and lysed by lysis buffer. RBP bound to MEG3 was captured by anti-Flag antibody affinity agarose beads; immunoprecipitation complexes were separated in 10\% SDS-PAGE gels and identified by specific antibodies.

(Fig. 6F). The protein components were determined by mass spectrometry, and the candidate proteins were confirmed by RNA pulldown and western blot analysis. Of the four candidate proteins selected in the co-expression network by GO analysis, ILF3 protein expression obviously increased, showing ILF3 is a MEG3-binding protein (Fig. 6G).

\section{Discussion}

DNA methylation is a major regulatory mechanism of tumor epigenetic control and it has an important role in gene transcription and splicing. It has been demonstrated that DNA promoter hypermethylation is associated with inactivation of several tumor suppressor genes, and these aberrant DNA methylations are always accompanied by a progressive dysregulation of DNMT expression (18). It is reported that the promoter region of lncRNA MEG3 is rich in $\mathrm{CpG}$ dinucleotides. The methylation pattern of the $2.1 \mathrm{~kb} 5$-flanking region of MEG3 contains functionally important sequences for gene expression (19). In general conditions, MEG3 is expressed in normal tissues, with high expression in the brain and pituitary gland (10). Many tumor tissues only express low MEG3, or even no MEG3 $(9,20)$. In pituitary tumors and leukemia, MEG3 promoter hypermethylation is also associated with the loss of MEG3 expression $(21,22)$. In our previous study, adenosine that has been confirmed to have cytotoxic effects on tumor cells also showed a demethylation role in HepG2 cells (15). In order to investigate the relationship between methylation status of MEG3 promoter and the expression of MEG3 gene, we treated hepatoma cells with adenosine or another selective DNMT inhibitor the 5-Aza-CdR. Our results showed that both adenosine and 5-Aza-CdR increased the expression of the unmethylated product and MEG3 mRNA expression (Fig. 1), thus revealing that MEG3 is correlated with a typical hypermethylation status in the promoter region in HepG 2 cells, and that we are the first to demonstrate that adenosine can trigger MEG3 activation. 
In order to further identify that MEG3 has an antitumor role, pcDNA3.1-MEG3 plasmid vectors were constructed and transfected into hepatoma cells. The results showed that ectopic expression of MEG3 inhibited cell growth in a timedependant manner (Fig. 3B) and increased cell apoptosis in HepG2 and Huh7 cell lines (Fig. 4A and C).

The cell cycle is divided into four distinct phases, G1, S, G2 and $\mathrm{M}$, and is tightly controlled by a series of key components including cyclins, the cyclin-dependent kinases. In this study, MEG3 induced cell cycle arrest significantly at the $\mathrm{S}$ phases and increased cell cycle arrest at the G0/G1 phases in HepG2 cells, accompanying by the decreased cyclin D1 expression, showing that cyclin D1 is essential for cell cycle transition of G1 to S phase and the decrease of cyclin D1 may lead to hampering cells from G0/G1 phase (23). However, we also observed that MEG3 caused different cell cycle arrest in two hepatoma cells (Fig. 4) and we considered the difference might be related to different p53 gene types, for HepG2 is a wild-type p53 and Huh7 is mutant p53 cell line.

Among all apoptosis-related genes, p53 is of particular importance (24). Tight regulation of the anti-proliferative activities of p53 is mostly achieved by the MDM2 ubiquitin ligase, which targets p53 for degradation (25). On the one hand, MDM2/p53 forms an autoregulatory feedback loop that may be needed to maintain a critical MDM2/p53 ratio within cells (26). On the other hand, an excess of MDM2 protein can abrogate the transcriptional activation of p53 (25). Factors that differentially regulate ubiquitination of MDM2 and p53 may affect cell fate profoundly as they can change the MDM2/ p53 ratio (27). Because wild-type p53 is a tumor suppressive factor while mutant p53 does not have this function, we chose wild-type p53 HepG2 cells for our research. Fig. 5 shows that ectopic expression of MEG3 decreased MDM2, and increased p53, and cleaved-caspase-3 expression in HepG2 cells, suggesting that downregulation of MDM2 contributes to p53 activation $(28,29)$. In addition, MEG3 might induce apoptosis through p53-independent pathways since exogenous MEG3 also inhibits Huh7 cell proliferation.

ILF3 is known to be a transcription factor and has two main isoforms (NF90 and NF110) (30,31). They participate in many aspects of RNA metabolism, including transcription, degradation and translation. In this study, an improved MS2-BioTRAP technique was used to screen the MEG3-binding proteins. Of the four candidate proteins selected in the co-expression network, ILF3 was identified as the binding protein by RIP, RNA pulldown and western blot analysis in HEK293 cells (Fig. 6). Zhu et al demonstrated that MEG3 can interact with DNA domain of p53 protein and regulate p53 target genes (32). Shamanna et al reported that knockdown of ILF3 by RNA interference led to elevated levels of p53 proteins in HPV-derived HeLa and SiHa cells (33). We speculated that MEG3 and ILF3 might form a complex which participates in the regulation of target genes (such as p53) and induce cell apoptosis. In this study, we observed that the expression level of ILF3 decreased after pcDNA3.1-MEG3 transfection in HepG2 cells. The mechanisms of ILF3 downregulation are due to increased ubiquitination and degradation, or other reasons that need to be further investigated.

In conclusion, this study demonstrates that the antitumor effect of adenosine is involved in MEG3 activation and the hypermethylation of MEG3 promoter region may contribute to its low expression. MEG3 negatively regulates hepatoma cell growth by affecting cell cycle progression and inducing cell apoptosis. Moreover, the present study identified that ILF3 is a MEG3 binding protein and might participate in the anticancer regulation of MEG3.

\section{Acknowledgements}

The authors are grateful for funding support from the National Nature Science Foundation of China (no. 30972925) and the Guangdong Natural Science Foundation in China (no. 2014A030313470). The authors would like to thank Professor En-min Li; and Dong-yang Huang and Yu-cai Fu for excellent technical support.

\section{References}

1. Bosetti C, Turati F and La Vecchia C: Hepatocellular carcinoma epidemiology. Best Pract Res Clin Gastroenterol 28: 753-770, 2014.

2. Ponting CP, Oliver PL and Reik W: Evolution and functions of long noncoding RNAs. Cell 136: 629-641, 2009.

3. Mitra SA, Mitra AP and Triche TJ: A central role for long noncoding RNA in cancer. Front Genet 3: 17, 2012.

4. Qin R, Chen Z, Ding Y, Hao J, Hu J and Guo F: Long non-coding RNA MEG3 inhibits the proliferation of cervical carcinoma cells through the induction of cell cycle arrest and apoptosis. Neoplasma 60: 486-492, 2013.

5. Yang Z, Zhou L, Wu LM, Lai MC, Xie HY, Zhang F and Zheng SS: Overexpression of long non-coding RNA HOTAIR predicts tumor recurrence in hepatocellular carcinoma patients following liver transplantation. Ann Surg Oncol 18: 1243-1250, 2011

6. Miyoshi N, Wagatsuma H, Wakana S, Shiroishi T, Nomura M, Aisaka K, Kohda T, Surani MA, Kaneko-Ishino T and Ishino F: Identification of an imprinted gene, Meg3/Gt12 and its human homologue MEG3, first mapped on mouse distal chromosome 12 and human chromosome 14q. Genes Cells 5: 211-220, 2000

7. Zhou Y, Zhong Y, Wang Y, Zhang X, Batista DL, Gejman R, Ansell PJ, Zhao J, Weng C and Klibanski A: Activation of p53 by MEG3 non-coding RNA. J Biol Chem 282: 24731-24742, 2007.

8. Zhou S, Wang J and Zhang Z: An emerging understanding of long noncoding RNAs in kidney cancer. J Cancer Res Clin Oncol 140: 1989-1995, 2014.

9. Zhou Y, Zhang X and Klibanski A: MEG3 noncoding RNA: A tumor suppressor. J Mol Endocrinol 48: R45-R53, 2012.

10. Zhang X, Zhou Y, Mehta KR, Danila DC, Scolavino S, Johnson SR and Klibanski A: A pituitary-derived MEG3 isoform functions as a growth suppressor in tumor cells. J Clin Endocrinol Metab 88: 5119-5126, 2003

11. Jia LF, Wei SB, Gan YH, Guo Y, Gong K, Mitchelson K, Cheng J and Yu GY: Expression, regulation and roles of miR-26a and MEG3 in tongue squamous cell carcinoma. Int J Cancer 135: 2282-2293, 2014

12. Kang W, Seol HJ, Seong DH, Kim J, Kim Y, Kim SU, Nam DH and Joo KM: Adenosine potentiates the therapeutic effects of neural stem cells expressing cytosine deaminase against metastatic brain tumors. Oncol Rep 30: 1101-1106, 2013.

13. Wu LF, Wei BL, Guo YT, Ye YQ, Li GP, Pu ZJ and Feng JL: Apoptosis induced by adenosine involves endoplasmic reticulum stress in EC109 cells. Int J Mol Med 30: 797-804, 2012.

14. Wu LF, Ye YQ, Huang GY, Li HB, Li GP, Pu ZJ, Wei BL and Feng JL: Involvement of endoplasmic reticulum stress in adenosine-induced human hepatoma HepG2 cell apoptosis. Oncol Rep 26: 73-79, 2011.

15. Xiang MQ, Liu LX, De W, Ye YQ, Pu ZJ and Wu LF: The mechanism of demethylation on adenosine and homocysteineinduced apoptosis in HepG2 cells. Chin Pharmacol Bull 30: 973-979, 2014.

16. Wu LF, Guo YT, Zhang QH, Xiang MQ, Deng W, Ye YQ, $\mathrm{Pu} \mathrm{ZJ}$, Feng JL and Huang GY: Enhanced antitumor effects of adenoviral-mediated siRNA against GRP78 gene on adenosineinduced apoptosis in human hepatoma HepG2 cells. Int J Mol Sci 15: $525-544,2014$ 
17. Gong C, Popp MW and Maquat LE: Biochemical analysis of long non-coding RNA-containing ribonucleoprotein complexes. Methods 58: 88-93, 2012

18. Lo PH, Tanikawa C, Katagiri T, Nakamura Y and Matsuda K: Identification of novel epigenetically inactivated gene PAMR 1 in breast carcinoma. Oncol Rep 33: 267-273, 2015.

19. Zhao J, Dahle D, Zhou Y, Zhang X and Klibanski A: Hypermethylation of the promoter region is associated with the loss of MEG3 gene expression in human pituitary tumors. J Clin Endocrinol Metab 90: 2179-2186, 2005.

20. Zhang X, Gejman R, Mahta A, Zhong Y, Rice KA, Zhou Y, Cheunsuchon P, Louis DN and Klibanski A: Maternally expressed gene 3, an imprinted noncoding RNA gene, is associated with meningioma pathogenesis and progression. Cancer Res 70: 2350-2358, 2010.

21. Gejman R, Batista DL, Zhong Y, Zhou Y, Zhang X, Swearingen B, Stratakis CA, Hedley-Whyte ET and Klibanski A: Selective loss of MEG3 expression and intergenic differentially methylated region hypermethylation in the MEG3/DLK1 locus in human clinically nonfunctioning pituitary adenomas. J Clin Endocrinol Metab 93: 4119-4125, 2008.

22. Benetatos L, Hatzimichael E, Dasoula A, Dranitsaris G, Tsiara S, Syrrou M, Georgiou I and Bourantas KL: CpG methylation analysis of the MEG3 and SNRPN imprinted genes in acute myeloid leukemia and myelodysplastic syndromes. Leuk Res 34: 148-153, 2010.

23. Shirali S, Aghaei M, Shabani M, Fathi M, Sohrabi M and Moeinifard M: Adenosine induces cell cycle arrest and apoptosis via cyclin D1/Cdk4 and Bcl-2/Bax pathways in human ovarian cancer cell line OVCAR-3. Tumour Biol 34: 1085-1095, 2013.

24. Shan X, Fu YS, Aziz F, Wang XQ, Yan Q and Liu JW: Ginsenoside $\operatorname{Rg} 3$ inhibits melanoma cell proliferation through down-regulation of histone deacetylase 3 (HDAC3) and increase of p53 acetylation. PLoS One 9: e115401, 2014.
25. Haupt Y, Maya R, Kazaz A and Oren M: Mdm2 promotes the rapid degradation of p53. Nature 387: 296-299, 1997.

26. Wu X, Bayle JH, Olson D and Levine AJ: The p53-mdm-2 autoregulatory feedback loop. Genes Dev 7A: 1126-1132, 1993.

27. Michael D and Oren M: The p53-Mdm2 module and the ubiquitin system. Semin Cancer Biol 13: 49-58, 2003.

28. Clegg HV, Itahana $\mathrm{Y}$, Itahana $\mathrm{K}$, Ramalingam $\mathrm{S}$ and Zhang $\mathrm{Y}$ : Mdm2 RING mutation enhances p53 transcriptional activity and p53-p300 interaction. PLoS One 7: e38212, 2012.

29. Lu KH, Li W, Liu XH, Sun M, Zhang ML, Wu WQ, Xie WP and Hou YY: Long non-coding RNA MEG3 inhibits NSCLC cells proliferation and induces apoptosis by affecting p53 expression. BMC Cancer 13: 461, 2013.

30. Monsalve M, Wu Z, Adelmant G, Puigserver P, Fan M and Spiegelman BM: Direct coupling of transcription and mRNA processing through the thermogenic coactivator PGC-1. Mol Cell 6: 307-316, 2000

31. Ohno M, Kunimoto M, Nishizuka M, Osada S and Imagawa M: $\mathrm{Ku}$ proteins function as corepressors to regulate farnesoid $\mathrm{X}$ receptor-mediated gene expression. Biochem Biophys Res Commun 390: 738-742, 2009.

32. Zhu J, Liu S, Ye F, Shen Y, Tie Y, Zhu J, Wei L, Jin Y, Fu H, Wu Y, et al: Long noncoding RNA MEG3 interacts with p53 protein and regulates partial p53 target genes in hepatoma cells. PLoS One 10: e0139790, 2015.

33. Shamanna RA, Hoque M, Pe'ery T and Mathews MB: Induction of p53, p21 and apoptosis by silencing the NF90/NF45 complex in human papilloma virus-transformed cervical carcinoma cells. Oncogene 32: 5176-5185, 2013. 